\title{
Offering Students a Selection of Multiple Class B/AB Power Amplifiers as a Remote Laboratory
}

\author{
http://dx.doi.org/10.3991/ijoe.v7iS1.1722 \\ Olaf H. Graven and Dag A. H. Samuelsen \\ Buskerud University College, Kongsberg, Norway
}

\begin{abstract}
In this paper a remote laboratory setup for conducting experiments on a class $\mathrm{B} / \mathrm{AB}$ power amplifier is presented, addressing the problems related to running experiments requiring temperature matching between transistors and bias diodes, in order to avoid thermal runaway in the transistors. In addition there is an option for the student to quickly examine a range of different transistors in both class $B$ and class $A B$ setups.
\end{abstract}

Index Terms—remote lab, class AB amplifier, class B amplifier, electrical engineering education

\section{INTRODUCTION}

Electrical engineering students complete a number of laboratory exercises during their degree program. The laboratory setup described in this paper is on class B and $\mathrm{AB}$ power amplifiers and is part of the second year module on analogue electronics.

Students attending a course in analogue electronics at the authors' university are required to perform a number of laboratory exercises involving different types of semiconductor circuits. Simple labs are done using breadboard and through-hole mounted components, but certain problems arises when setting up a laboratory exercise involving the very commonly used class $\mathrm{AB}$ power amplifier. The main problem is that the amplifier requires that the bias diodes and the transistors share a common heat sink, as the temperature characteristic of transistors cause the transistor to start conducting at lower base-emitter voltage levels as the junction temperature increases. As the baseemitter voltage is supplied by the bias network, the bias voltage must be reduced accordingly, and this is done by letting the bias diodes experience the same temperature change as the transistors.

It is difficult to simulate amplifier circuits accurately in circuits where temperature changes affect the bias point of the transistors. The only solution to this is to do the experiment on real physical hardware.

Given these challenges on the circuit creation two alternatives for the experiment presents itself: Manufacture a class set of physical boards for the students, with the cost and maintenance associated. Or, create one setup and make it available for the students remotely. The remote setup does require some extra initial design and development work, especially to allow the students a wide range of setup choices. But the remote setup does offer a simpler and cheaper maintenance and a possibility for the students to access the laboratory $24 / 7$, and not be dependent on the limited opening hours of the physical laboratories.

The purpose of this laboratory exercise is to give the students a thorough understanding both class B and class
$\mathrm{AB}$ and the difference between them. The main challenge in running the lab in the traditional way is that students often fail to make the connections right the first time, and hence, waste a lot of time on troubleshooting the circuit. It is also quite difficult for the students to keep focus both on the results they are getting, and at the same time do connections right, take notes, use the measurement instruments correct, and set the inputs to the system. It is the authors' experience that all the side activities contribute to obfuscating the real aim of the lab: understanding the circuits. There is therefore a desire among the staff to separate the training in using the breadboard to create electrical circuits, from the understanding gained in the laboratory exercise.

The remote laboratory solution presented in this paper removes the breadboard from the students replacing it with a web interface to the laboratory, while keeping the physical part of the laboratory.

\section{Simulations AS AN Alternative}

An alternative to a remote laboratory setup could be a normal simulation program to run on a PC, but several disadvantages have been identified to such a solution, given these challenges:

- As mentioned previously, it is difficult to simulate amplifier circuits accurately in circuits where temperature changes affect the bias point of the transistors. This is in particular true for the class $A B$ power amplifier, requiring close temperature match between the transistors and the diodes in the bias network. The diodes in this type of amplifier are mounted on the same heat sink as the transistors in order to avoid thermal runaway caused by the reduced voltage drop in the base-emitter part of the transistors when the substrate temperature rises, as the voltage from the bias network otherwise would be constant.

- A simulation will always be an approximation to the real world, and any property of the physical machine not included in the model, will add to the discrepancy between the real world and the simulation.

- Noise components could be artificially added to a simulation, but noise is also a model of the real world noise, and may not entirely correct imitate the real world noise.

- If a good simulation could be created, it would require huge computational resources, and it may take from many minutes to hours for the simulation model to reach stationary values. With a remote lab setup as the one presented in this article, the results are present after a second, always. 


\section{A. The setup of an remote laboratory}

The use of web-based laboratory is not new, and there has been implemented similar projects using a number of different development tools and platforms. These include among others: Casini et.al [4] with Matlab, Das et.al [5] with Visual Basic, Pradarelli et.al. [6], and Ferreira and Muller [7] using a home developed systems. The main difference between previous setups and the one presented in this paper is the focus on aiding the students understanding of the theory and not focusing on making a copy of a physical laboratory, the ease of implementation, and low cost of the system in this article,. Using standard lowcost acquisition devices from National Instruments, together with a standard PC, makes the implementation doable on a very low budget.

The presented remote laboratory does not facilitate students who prefer to touch and handle the equipment themselves. This is however not regarded as a serious problem, as the module in analogue electronics that use this remote experiment requires the students to complete other real life in the laboratory experiments.

\section{B. Lifelike remote interface}

A lot of remote experiments focus on creating an as lifelike as possible interface [8,9]. This includes: Using pictures or graphical representations as close to the real laboratory equipment as possible. Having students click on pictures of terminals to connect up the equipment to measuring points. Using a live webcam of the circuit, this most of the time just shows the circuit with LED's if it is a digital and just the circuit if it is an analogue. This webcam is presumable used to ensure to the students that there really is a circuit and that it is not smoking.

While the approach of making the experiment as lifelike as possible have some merit when the remote laboratory are the only connection the students get to laboratories, the authors feel that this approach is not required in the experiment presented in this paper. The reason being, that the students in the group using the remote laboratory experiment presented here are required to complete a number of real hands on experiments in a laboratory. Thus in the authors' opinion removing the need for a look and feel like the real world, this then gives us an opportunity to design the graphical user interface with a focus on aiding the students understanding.

The approach favoured in this paper, where the graphical user interface is designed not to look lifelike but rather focuses on ease of use, may at first seem strange. The rationale for this approach is simple, from observing students over a number of years it has for the authors become apparent that presenting students with multiple learning challenges simultaneously does not work well. The challenges in this laboratory experiment would be the use of complex laboratory equipment and understanding the theory behind a power amplifier circuit.

The argument often put forward against this approach is often, (these are quotes from some of our colleges): "They need to learn to use the laboratory equipment so it would be easier to learn it at the same time." or "we managed to learn it this way", or even "we have taught the students this way for $x$ years and it has always worked well", substitute between 10 and 40 for $\mathrm{x}$, any figure that are appropriate to the person. Our argument is, and we feel that more should adhere this is that, just because you suffered does not mean that your students should.

In addition to the basic theories and other material the students have to understand there are also an ever increasing amount of new and occasionally complex tools and equipment engineering students need to learn and master. We feel that our approach where we separate out some of these learning objectives and create laboratory exercises that focus on a few or even just a single learning objective at a time, the students can focus on that and not be overloaded with new areas to master simultaneously.

Our approach is to divide the learning objectives into different categories and handle them separately. One category for understanding the theory behind a power amplifier (the topic for this paper) and another for learning to master the equipment in the laboratory, do in addition to aiding the student when they are in the process of learning the material, also allow us to direct students, or hopefully they can themselves redo the work on the learning objectives they are still unable to master. The mastering of the laboratory equipment is either done on simple circuits that are well understood or they can be done on more complex circuits for which the students have first received a thorough theoretical background. By diving up the learning objectives in such a way it is the authors belief that we may wake an intrinsic motivation in the students, where they are able to succeed in completing the tasks put in front of them, and the completion of the task itself becomes the reward and a motivation to keep going. Such a state is well know from learning theory and is known as flow. [10]

The authors want to emphasize here that our aim is on creating an environment where the students' learning process have the focus. It is the students who need to learn and understand the material, and hence, have to put in the work. So our means of reaching our aim is to create a state of flow in the students where the completion of a piece of work itself is the reward and the motivation to keep going.

\section{The DESIGN AND CONSTRUCTION OF THE REMOTE LABORATORY}

\section{A. The remote laboratory setup}

The remote laboratory is constructed using a software part and a hardware part. The software part is realized using National Instrument's LabVIEW, with remote panels and web server. The hardware consists of the amplifier circuit for running the experiment, and the support system for this circuit. The support system is the main focus of this section, and has the following tasks:

- Generation of signals for the amplifier input.

- Measurement of voltages and currents, with buffers between the point of measurement and the acquisition card.

- Allows the user to remotely change components and component values, as well as to change the transistor type and the topology of the amplifier network.

- Apply varying degree of cooling to the heat sink for demonstrating the effect of varying junction temperature on the bias point.

- Protection and supervision of the amplifier and the components. 
The input of the amplifier receives a signal from the analogue output of the acquisition board. This signal is generated by the LabVIEW software and is sinusoidal with amplitude and frequency set by the user. In this way, the frequency response of the amplifier can be determined for the various configurations of the input and output circuit, and the effect on signal distortion and heat generation of small and large signals, related to the loading of the amplifier through the load resistor.

Measurements are done using the analogue inputs of the acquisition card. The maximum voltage on these inputs is $\pm 10 \mathrm{~V}$, with reference to system ground. Normally, this type of amplifiers uses a power supply of $\pm 15 \mathrm{~V}$ to $\pm 60 \mathrm{~V}$. Voltage dividers are therefore required between some of the measurement points and the analogue inputs. These are made from precision resistors in order to keep a certain level of accuracy. This, however, introduces a new problem: Even though the input bias current on the analogue inputs is negligible, the acquisition card (due to the low cost) only have one analogue to digital converter (ADC), and use a multiplexer to select one of the analogue inputs. The stray capacitance of this multiplexer cause a relatively large current to flow in or out of the analogue input in the few microseconds it takes to change the voltage between inputs, meaning the source of the measurement should have a very low output resistance, while the measurement points should have a negligible effect on the circuit's performance. The solution to this problem is to install operational amplifiers configured as voltage followers between the voltage divider and the acquisition card.

For changing resistor values, analogue solid state switches [1] or multiplexers [2], are used in combination with external resistors. These elements have relatively low switch resistance $(<100 \Omega)$, low internal capacitance $(<200 \mathrm{pF}$, combined), and good matching between components (typical 5\%). Using these elements, the standard E12 series (or any other series) of resistor values can be set up. An identical approach is used for capacitors, except for the high current paths.

The class B and AB amplifier have approximately unity voltage gain. The current gain is usually much larger. This means that the current flowing in the output of the amplifier usually is significantly larger than the input currents. This has an impact on the use of switching elements. The solid state switches described above have a typical onresistance of $50 \Omega$. When the equivalent of a typical load

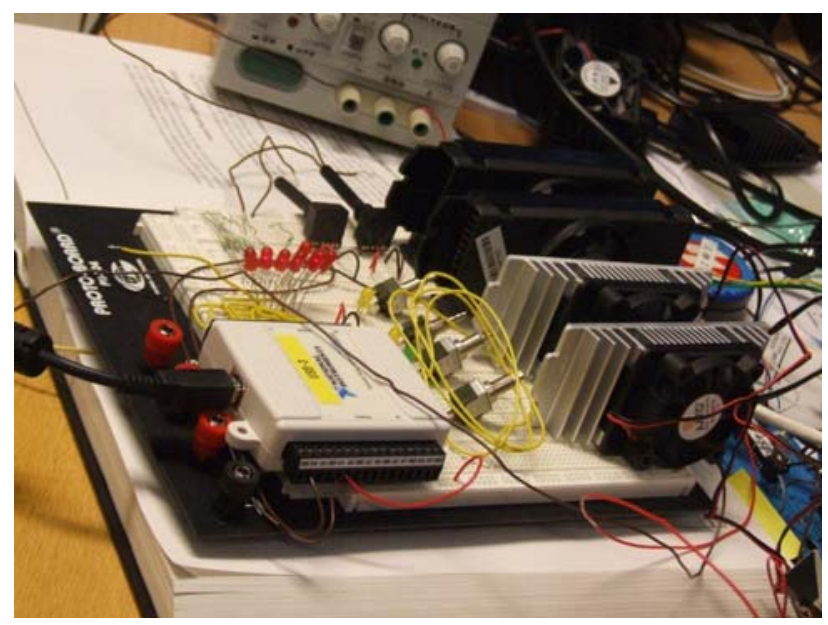

Figure 1. Hardware setup of the heat-sinks for the transistors and the diodes, and the measurement system.

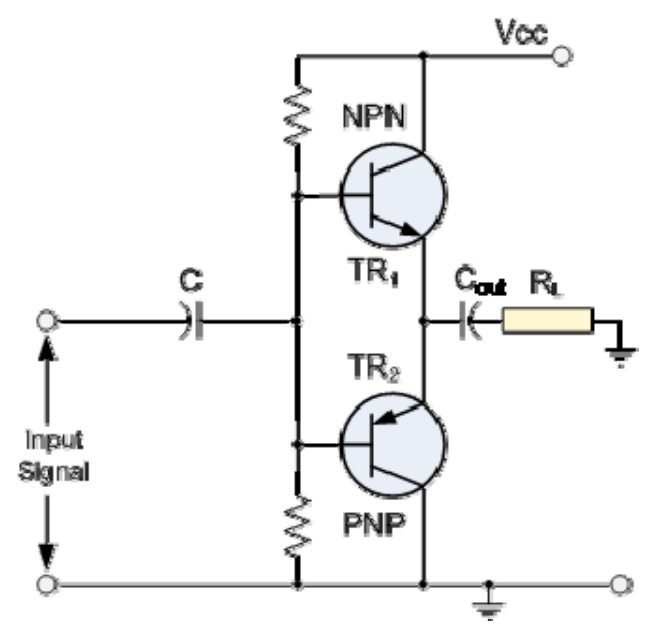

Figure 2. Basic circuit of class B power amplifier with single power supply.

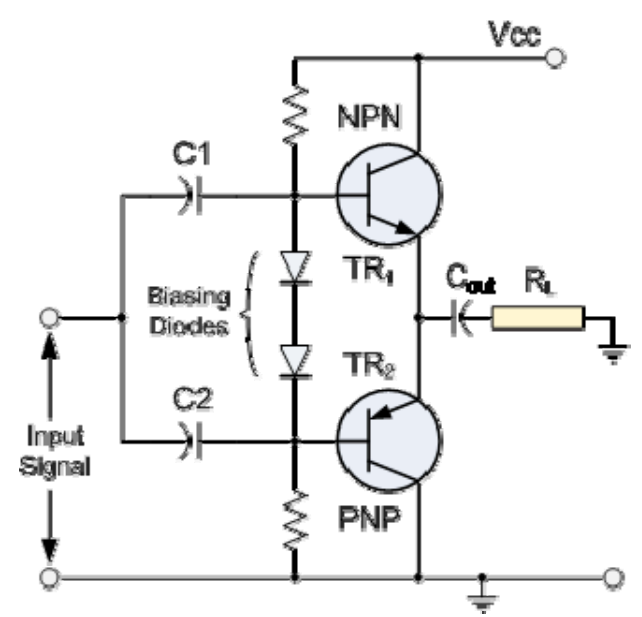

Figure 3. Basic circuit of class AB power amplifier with single power supply.

resistor has a value between $4 \Omega$ and $32 \Omega$, this gives that the added voltage drop in the switch would be unrealistic high. Instead, relays are used for connecting and disconnecting components.

In addition to the buffer and snubber circuits, a memory element must be installed for each relay, due to the limited number of digital $\mathrm{I} / \mathrm{O}$ lines on the card. These memory elements (usually D-flip-flops) are accessed using a simple bus system with separate data, address and control bus, allowing for a large number of switching elements in the experiment, giving the users more options in choosing components for the circuit.

The two amplifier circuits are shown in figure 1 and 2 . In order to avoid thermal runaway in the transistors, the diodes in the bias circuit of the class $A B$ amplifier is mounted close to the transistors, sharing heat sink with the transistors. This reduces the risk that the negative temperature coefficient of the base-emitter junction does not lead to over-currents, as the bias network have the same negative temperature coefficient, experiencing the same temperature change as the transistors. In addition, the heat sink is fitted with fans which allows for forced airflow over the heat sink. This gives the option of controlling the temperature of the transistors, and allows the users to see what effect temperature change has on the bias voltages in the amplifier. 
In order to make the system foolproof, an extra layer of supervision and protection is implemented. If a state of over-current or over-temperature occur that might cause permanent damage on the components, the support system is set to disconnect the power supply, until the error state is rectified.

\section{B. Options when using the software}

The graphical user interface controlling the remote laboratory is fairly simple in a setup like the one described in this paper. To give the students as much flexibility as possible the user input is divided into two phases:

1. Set all the values. This means setting the values for amplitude and frequency for the input signal. Selecting the type of circuit to use, class $\mathrm{AB}$ or a pure class $\mathrm{B}$ amplifier. In addition to selecting the class of amplifier there are also several sets of class $A B$ and class B setups. Then choosing the values for the resistors and capacitors for the bias circuit. Finally choosing the resistance for the load. The setting of these values can be done in any order and changed as many times as s desired.

2. Choose and re-choose at will which measurements to view and in what combination.

The first part is similar to what can be done in a normal laboratory, the simulations setup and use of multiple class $\mathrm{B}$ or class $\mathrm{AB}$ circuits can be both cumbersome and require a lot of effort. This option has been prearranged for the users and their work is reduced to only selecting one of a range, the go back and select another pair to see the difference characteristics different "similar" transistor can give.

The second part is where the experiment start to differ significantly from what can be done in a physical laboratory. After the experiment is run and the application has registered all the measurements, the students are free to display whichever measurements in any combination they desire. Giving the students the opportunity to select what measurements to display after experiment is run, is a conscious decision by the authors. It can be argued that making the students think through their setup before running the experiment is more in line with the real world, but it is the authors opinion that giving the students the opportunity to select measurements for display and easily changing their selection it would aid the students in "playing", with the circuit and thereby again aiding their understanding.

The graphs used to display the results are standard graphs included with LabVIEW. These graphs offer a multitude of options for the students on zooming by selecting a range of data, thus giving them a clearer view of signals. The students can also zoom in on the level of values to gain an accurate reading.

\section{Assignment description}

Giving the students a thorough understanding of how electronic circuits actually work is the main objective of the assignment. The ultimate goal is for the students to achieve an intuitive feel for how a certain circuit would behave when subjected to an input signal. There are several types of training used in achieving this goal, and a student should be subjected to several training elements rather than focusing on one element alone. This makes it important to be able to adapt the amount of training to each student's need.

The theoretical background will always play an important role in this type of modules, and is usually the first part done in the classes. For the students to move further on, practical experience from laboratories and simulations are used for the true complex nature of the semiconductor circuits to be revealed.

The theoretical background for doing calculations on electronic circuits is presented for the students, before running simulations and practical experiments. This gives the students an opportunity to see the difference between a calculated behaviour, a simulated result and a practical result. Calculations are done using very simplified models, taking into account only the most important properties of the circuit's behaviour. There will always be a difference between the calculated results and the simulated results, due to the far more complex models used in simulations. The problem with the class $A B$ power amplifier is the great difficulty in achieving realistic simulations due to the tight relations between the electrical and the thermal behaviour of this type of circuit. This causes a significant difference between the results from simulations and practical trials.

Other aspects that can be investigated by the students are the effect on frequency response the different combinations of input/output capacitor and bias resistors have, the difference between the class $\mathrm{B}$ and class $\mathrm{AB}$ amplifier with respect to the crossover distortion, and how well the biasing of the transistors reduces the distortion, as well as how the loading of the different amplifier topologies affect the thermal behaviour.

A web interface is used to allow the students access to this laboratory, and an assignment is usually carried out by the following steps:

- Preliminary calculations on the circuit are performed to find a starting point for component values in the circuit.

- The circuit topology is selected based on the assignment.

- Component values are selected based on the previous calculations.

- The circuit is run with the selected components and a predefined signal and load.

- After inspection of the graphs from the trial, component values might be changed and the circuit can be run over again.

In addition to allowing the students to run experiments on this type of circuits, this also demonstrates how easy and quick the trials can be done, without wasting time and cognitive resources on doing connections, troubleshooting, etc.

\section{CONCLUSION}

We have here shown an implementation of a remote laboratory for class $\mathrm{B}$ and $\mathrm{AB}$ power amplifiers. As the remote lab is done on real, physical components, the results given are realistic, and the difference between a simulation and a real experiment can be identified.

The laboratory described address some the challenges faced with running a student laboratory with power amplifiers. We have addressed the challenge of negative tem- 
perature coefficient in the transistor, which is not correctly modelled in a normal simulation. It is even possible to actually change the topology of the circuit remotely, allowing the students to easy see the difference between different amplifier topologies. We have given the students a laboratory that they are able to utilize and do aid in their understanding of the theory better than a traditional lab. The authors are aware that offering a remote rather than a traditional physical laboratory has its drawbacks, but these are addressed by the fact that this laboratory is a part of a set of laboratories run in the module covering analogue electronics.

The remote laboratory has been implemented using relatively low cost components. The development time spent on the laboratory itself was within a reasonable short time frame, about a week full time effort. The result is a fully automated remote laboratory that runs 24/7 without any interaction apart from the students using it. This gives the students the opportunity of doing the assignments at the time and place of their choice, which is useful when students have competing activities, besides the study.

\section{REFERENCES}

[1] Maxim IC, Maxim IC, Application note 638: "Selecting the right CMOS analog switch", retrieved from: http://pdfserv.maximic.com/en/an/AN638.pdf, 01.08.2010.

[2] Intersil, Intersil, Datasheet FN3116.9: "DG406, DG407", retrieved from http://www.intersil.com/data/fn/fn3116.pdf, 01.08.2010.

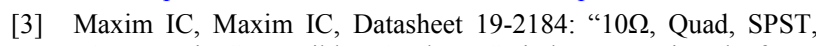
+3V Logic-Compatible Analog Switches", retrieved from: http://datasheets.maxim-ic.com/en/ds/MAX312-MAX314.pdf, 01.08 .2010
[4] M. Casini, D. Prattichizzo, and A. Vicino, "The automatic control telelab: a user-friendly interface for distance learning," vol. 46, no. 2, pp. 252-257, 2003.

[5] S.L.G.A.Das,S.,"Remote communication engineering experiments through internet," iJOE International Journal on Online Engineering, 2006.

[6] B. Pradarelli, L. Latorre, and P. Nouet, "Integrated circuits testing: Remote access to test equipment for labs and engineering," iJOE, vol. 5, Special Issue 1: REV2009, 2009.

[7] J. M. M. Ferreira and D. Mueller, "Online labs and the marvel experience," iJOE, vol. 1, No.1, 2005.

[8] Zorica Nedic, Jan Machotka, and Andrew Nafalski, "Remote Laboratory NetLab for Effective Interaction with Real Equipment over the Internet" HSI 2008, IEEE, Krakkov Polen, 2008.

[9] Ingvar Gustavsson, Thomas Olsson, Henrik Åkesson, Johan Zackrisson, Lars Håkansson, "A Remote Electronics Laboratory for Physical Experiments using Virtual Breadboards" 2005 American Society for Engineering Education Annual Conference \& Exposition, American Society for Engineering Education, 2005.

[10] M. Csikszentmihalyi and I. Csikszentmihalyi, Beyond boredom and anxiety. San Francisco ,: Jossey-Bass, 1975.

\section{AUTHORS}

O. H. Graven is with Buskerud University College, Department for Technology, Kongsberg, Norway (e-mail: olaf.hallan.graven@hibu.no).

D. A. H. Samuelsen is with Buskerud University College, Department for Technology, Kongsberg, Norway (email: dag.samuelsen@hibu.no).

This article is an extended version of a paper presented at the International Conference EDUCON2011, held in April 2011 at PSUT, in Amman, Jordan. Received June $25^{\text {th }}, 2011$. Published as resubmitted by the authors August $2^{\text {nd }}, 2011$. 